\title{
Millimeter and submillimeter wave spectroscopy of HNC and DNC in the vibrationally excited states
}

\author{
Toshiaki Okabayashi and Mitsutoshi Tanimoto \\ Department of Chemistry, Faculty of Science, Shizuoka University, 836 Oya, Shizuoka 422, Japan
}

(Received 31 March 1993; accepted 24 May 1993)

\begin{abstract}
The rotational transitions of hydrogen isocyanide (HNC) and deuterium isocyanide (DNC) in the vibrationally excited states as well as in the ground states were observed in the millimeter and submillimeter wave region. These compounds were generated in a dc glow discharge plasma containing hydrogen (or deuterium), nitrogen, and carbon atoms. The stretching vibrational modes, $v_{1}$ and $v_{3}$ states, were selectively excited in the discharge plasma; on the other hand, the bending mode $v_{2}$ state was thermally populated at the cell temperature. The precise rotational, centrifugal distortion and $l$-type doubling constants were obtained for all of the first vibrationally excited states as well as the ground states. The experimental equilibrium rotational constants $B_{c}$ are $45496.7769(45)$ and $38207.7217(105) \mathrm{MHz}$ for HNC and DNC, respectively, where uncertainties correspond to one standard deviation. The equilibrium internuclear distances are also determined to be $r_{e}(\mathrm{H}-\mathrm{N})=0.9960643(29) \AA$ and $r_{e}(\mathrm{~N} \equiv \mathrm{C})=1.1683506(16) \AA$.
\end{abstract}

\section{INTRODUCTION}

Since the first detection of an interstellar millimeter wave emission line U90.7, ${ }^{1,2}$ which has subsequently been assigned to the $J=0-1$ transition of the HNC molecule, microwave studies have been performed on $\mathrm{HNC}$ and its isotopic species DNC in several laboratories. Creswell and his co-workers ${ }^{3,4}$ observed the rotational transitions in the ground vibrational state of not only the parent but also the rare isotopic species in the products of the reaction of methyl iodide with excited nitrogen and determined the substitution molecular structure. Saykally et al. ${ }^{5}$ independently observed the $J=1-0$ transition of HNC in de glow discharges through mixtures of cyanogen and hydrogen, cyanogen and acetylene, or nitrogen and acetylene. Blackman et al. ${ }^{6}$ also mcasured the $J=1-0$ lines of HNC and DNC generated by heating samples of $\mathrm{HCN}$ or DCN to temperatures of about $1000 \mathrm{~K}$, and determined the electric dipole moment (3.05 \pm 0.1 Debye) from Stark effect measurements. Recently, Woods ${ }^{7}$ reported the experimental equilibrium structure of HNC.

In the infrared region the first observation was reported by Milligan and $\mathrm{Jacox}^{8,9}$ following the photolysis of $\mathrm{CH}_{3} \mathrm{~N}_{3}$ or $\mathrm{HCN}$ in an Ar matrix at $4 \mathrm{~K}$. The vibrationalrotational spectrum of the $v_{1}$ fundamental band of gaseous HNC was observed in emission in the reaction of active nitrogen with various molcculcs by Arrington and Ogry$z_{10}{ }^{10}$ with a low resolution spectrometer. Maki and Sams ${ }^{11}$ measured with a high resolution grating spectrometer not only the $v_{1}$ bands but also accompanying $v_{2}$ hot bands of HNC and DNC in equilibrium with HCN (or DCN) at high temperature. The most detailed study of HNC, which was generated by reacting the products of a microwave discharge of $\mathrm{N}_{2}$ with $\mathrm{CH}_{3} \mathrm{Br}$, was carried out by Burkholder et al. ${ }^{12}$ with a high resolution Fourier transform infrared (FTIR) spectrometer. They observed the $v_{1}$, $v_{2}$, and $v_{3}$ fundamental bands and determined accurate molecular constants. However there has been reported no spectroscopic data for the $v_{3}$ state of DNC.

The present paper reports measurements of the rotational transitions in all of the first excited states as well as in the ground state of HNC and DNC in the millimeter and submillimeter wave region. The observed rotational spectra were analyzed by the least-squares method for each vibrational state to obtain precise values of the rotational, centrifugal distortion, and $l$-type doubling constants. Using the rotational constants thus obtained, we determined the vibration-rotation constants and the equilibrium rotational constants of HNC and DNC. Moreover, we also determined the equilibrium structure from two equilibrium rotational constants.

\section{INSTRUMENT}

Figure 1 shows a schematic diagram of the microwave spectrometer newly constructed in Shizuoka University and used in the present experiment. As microwave sources, OKI millimeter wave klystrons are employed in the region below $130 \mathrm{GHz}$. The millimeter wave radiation in the 200 to $280 \mathrm{GHz}$ region is generated by multiplying the output of a klystron using a tripler (Millitech MU-3-03-T1). The tripler also provides spurious fourth harmonic power which is adequate as the source in the submillimeter wave region above $300 \mathrm{GHz}$. The microwave transmitted through a free space cell is detected by a liquid-He cooled InSb hot electron detector (QMC QFI/2).

The millimeter wave klystron is repetitively scanned in frequency by applying a sawtooth of $10 \mathrm{~Hz}$ to its reflector electrode. The frequency of the klystron is monitored by referring to harmonics of an X-13 centimeter wave klystron, which is phase locked by a microwave stabilizer (Dymec DY2650A) to the output of a synthesized signal generator (HP8656A). A microwave counter (Advantest R5372) which counts the $X-13$ frequency and the signal generator are controlled by a 16-bit personal computer 


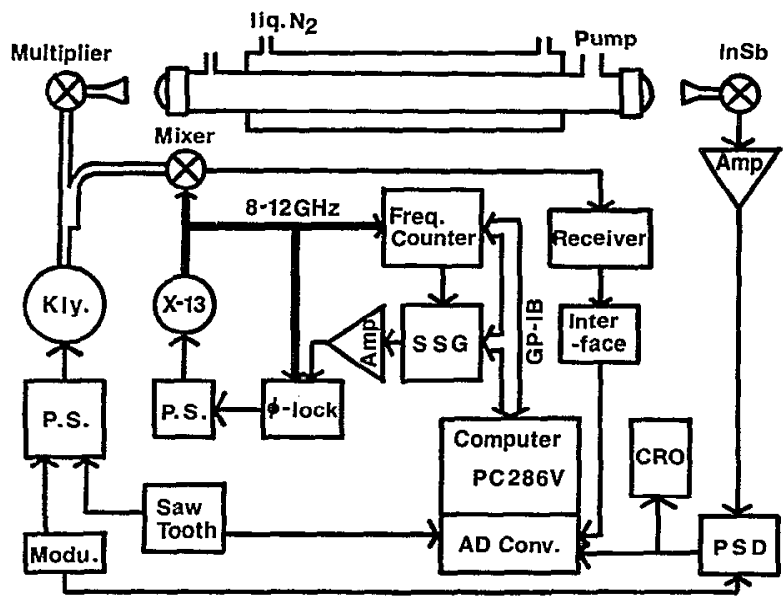

FIG. 1. A schematic diagram of the microwave spectrometer.

(EPSON PC286V) through a GPIB interface. Beat notes between the output of two klystrons are detected by a radio-frequency receiver (Nissin Electronic NRR-201A).

The microwave is frequency modulated to a full width of a few hundred $\mathrm{kHz}$ by applying a bipolar square wave of $50 \mathrm{kHz}$ to the reflector of the millimeter wave klystron. A phase sensitive detector (NF LI-517) is operated at 100 $\mathrm{kHz}$ with $1.25 \mathrm{~ms}$ time constant, and the recorded signal had a second derivative lineshape.

The PSD output, the shaped beat notes from the receiver, and the monitor signal from the sawtooth are fed into a 12 bit $\mathbf{A} / \mathbf{D}$ converter. After digitization, the PSD output is allocated to 256 data points for every scan, the start and end of which are decided by voltages of the sawtooth monitor signal. The positions of a plus and a minus beat note are employed to calculate the frequency scale for the absorption signal by interpolation. The process is repeated for every scan to accumulate for several minutes. The computer software is mainly written in BASIC language and partly complemented by $\mathrm{C}$ language which is suitable for controlling hardware.

The free-space-type absorption cell is of $2 \mathrm{~m}$ long and $10 \mathrm{~cm}$ o.d. Pyrex glass tube, sealed with a pair of Teflon lenses of the focal length of about $25 \mathrm{~cm}$ at both ends. The cell is evacuated by a mechanical booster pump (Shinko Seiki) of $2500 \mathrm{l} / \mathrm{min}$. A pair of cylindrical stainless steel electrodes are inserted at each end of the cell for discharge. The cell is surrounded by a cooling jacket made of copper with a soldered pipe through which liquid nitrogen is circulated. Adjustment of the rate of the liquid nitrogen flow can control the cell temperature to the desired value between $77 \mathrm{~K}$ and room temperature. The cell is installed in a thick styrofoam block for thermal insulation.

\section{EXPERIMENT}

HNC and DNC molecules were directly generated in the absorption cell by a dc glow discharge. In order to avoid interfering lines originating from starting materials or generated by-products, we employed several reaction systems:

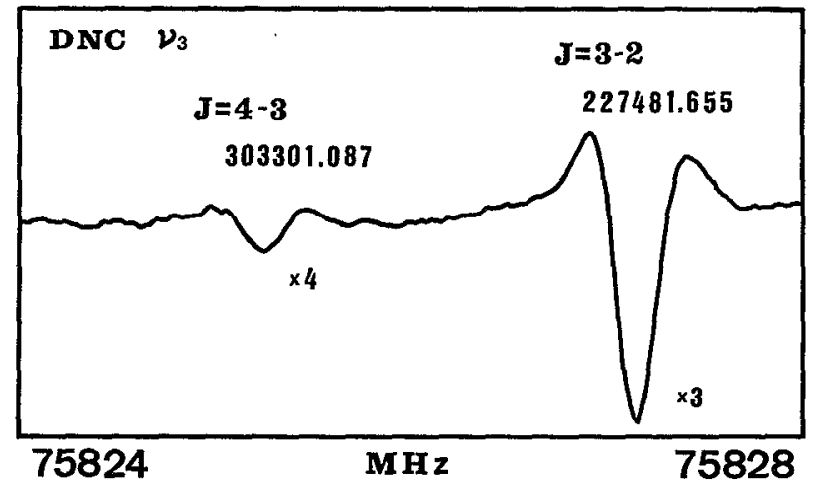

FIG. 2. The millimeter and submillimeter wave spectra of rotational transitions $J=3-2$ and $J=4-3$ in the $v_{3}$ excited state of DNC. The horizontal axis represents the klystron fundamental frequency. The frequency given over the absorption lines is the product of the klystron frequency and harmonics which is also indicated. The signal was accumulated for $10 \mathrm{~s}$.

for $\mathrm{HNC}$

$$
\begin{aligned}
& \mathrm{H}_{2} \mathrm{C}=\mathrm{CHCN} \text { (20 mTorr) }+\mathrm{H}_{2} \text { (10 mTorr) } \\
& +\mathrm{He} \text { (30 mTorr), } \\
& \mathrm{CH}_{3} \mathrm{CN} \text { (30 mTorr) }+\mathrm{He} \text { (30 mTorr), }
\end{aligned}
$$

or

$$
\mathrm{CH}_{3} \mathrm{I} \text { (25 mTorr) }+\mathrm{N}_{2} \text { (25 mTorr) }
$$

and for $\mathrm{DNC}$

$$
\mathrm{CD}_{3} \mathrm{CN} \text { (30 mTorr) +He (30 mTorr), }
$$

or

$$
\mathrm{CD}_{3} \mathrm{I} \text {. (25 mTorr) }+\mathrm{N}_{2} \text { (25 mTorr). }
$$

We mainly chose the (A), (B), or (D) reaction systems because in these systems the line shape of the target molecule spectrum was narrower and there were less interfering lines than in the $(C)$ or $(E)$ reaction systems. The generation condition was determined by monitoring the line intensity in the ground state. The discharge current was adjusted around $300 \mathrm{~mA}$ and the temperature of the cell was kept at around $210 \mathrm{~K}$. The line strength of HNC and DNC lines was not sensitive to the discharge conditions, such as the cell temperature, the discharge current and the sample pressure.

Based on the prediction from the infrared result, ${ }^{11,12}$ we readily observed the rotational transitions in the $v_{1}, v_{2}$, and $v_{3}$ excited states of $\mathrm{HNC}$, and in the $v_{1}$ and $v_{2}$ states of DNC. The transition frequencies of DNC in the $v_{3}$ state were predicted by assuming that the vibration-rotation constant $\alpha_{3}$ is equal to that of $\mathrm{DNN}^{+}(239 \mathrm{MHz}),{ }^{13}$ since $\mathrm{DNC}$ and $\mathrm{DNN}^{+}$have same electronic structure and similar rotational constants in the ground state (38 $152 \mathrm{MHz}$ and $38554 \mathrm{MHz}$, respectively). We searched for the $v_{3}$ lines on a cathode ray oscilloscope and easily detected them at the position of $\alpha_{3}=238 \mathrm{MHz}$ (Fig. 2).

Under the experimental conditions described above, the line intensities of the $v_{1}$ and $v_{3}$ states were found to be 
TABLE I. Observed transitions of HNC and DNC (MHz). ${ }^{\mathrm{a}}$

\begin{tabular}{lccll}
\hline \hline State & $J=1-0$ & $J=2-1$ & $J=3-2$ & $J=4-3$ \\
\hline HNC & & & & \\
Ground & $90663.593(9)^{\mathrm{b}}$ & $181324.758(-8)^{\mathrm{b}}$ & $271981.142(2)$ & $362630.303(0)$ \\
$v_{1}$ & & & $270185.386(-20)$ & $360236.110(15)$ \\
$v_{2}(e)$ & $\ldots$ & $271924.156(-13)$ & $362554.351(9)$ \\
$\quad(f)$ & $\cdots$ & $273869.532(-13)$ & $365147.495(9)$ \\
$v_{3}$ & $89993.197(12)$ & & $269969.964(-9)$ & $359948.787(3)$ \\
DNC & & & & \\
Ground & $76305.727(7)^{\mathrm{b}}$ & $152609.774(1)^{\mathrm{b}}$ & $228910.489(-6)$ & - \\
$v_{1}$ & $75704.545(38)^{\mathrm{d}}$ & & $227106.935(-13)$ & $305206.219(2)$ \\
$v_{2}(e)$ & $\ldots$ & $229327.206[50]^{\mathrm{c}}$ & $302801.600(5)$ \\
$\quad(f)$ & $\ldots$ & $231070.095[50]^{\mathrm{c}}$ & $308084.675[50]^{\mathrm{c}}$ \\
$v_{3}$ & $75829.460(11)$ & $227481.655(-8)$ & $303301.087(3)$ \\
\hline \hline
\end{tabular}

${ }^{a}$ Values in parentheses represent the residual (Obs.-Calc.) in $\mathrm{kHz}$.

${ }^{\text {b} F r o m ~ R e f s . ~} 3$ and 4.

${ }^{c}$ Estimated experimental error.

${ }^{d}$ Less weighted because of poor $\mathrm{S} / \mathrm{N}$ ratio.

abnormally strong. The effective vibrational temperature was estimated from the line intensities; $1300-1600 \mathrm{~K}$ for the $v_{1}$ and $v_{3}$ states and $230 \mathrm{~K}$ for the $v_{2}$ state. This means that the stretching mode was selectively excited in the discharge plasma, and that the bending mode, on the other hand, was thermally populated.

\section{ANALYSIS}

Table I lists the measured rotational transitions between $J=1-0$ and $J=4-3$ for two isotopomers. The observed spectral lines were analyzed using the standard rotational energy formula for a linear molecule:

$$
\begin{aligned}
E_{v, J}= & B_{v}\left[J(J+1)-l^{2}\right]-D_{v}\left[J(J+1)-l^{2}\right]^{2} \\
& \pm \frac{1}{2}\left[q+q_{J} J(J+1)\right] J(J+1),
\end{aligned}
$$

where $v$ and $l$ are the quantum numbers of the vibrations. The value of $l$ is fixed to one in the $v_{2}$ state and to zero in the other states. The term of $q$ and $q_{J}$ in Eq. (1) accounts for the $l$-type doubling in the $v_{2}$ excited state with the + and - signs for $f$ and $e$ state, respectively.

The transition frequencies were subjected to leastsquares analysis to give the molecular constants summarized in Table II. The observed transitions in the $v_{2}$ state of DNC are so limited that we could not determine the molecular constants accurately. The present molecular constants are in good agreement with those of the previous infrared work ${ }^{11,12}$ but are more precise.

\section{DISCUSSION}

The stretching vibrational modes of $\mathrm{HNC}$, the $v_{1}$ and $v_{3}$ states, were selectively excited in the discharge plasma. The phenomenon like this case has been reported; for HBO (Refs. 14 and 15) in the discharge plasma of $\mathrm{B}_{2} \mathrm{H}_{6}, \mathrm{O}_{2}$, and $\mathrm{He}$ mixture, for $\mathrm{HCO}^{+}$(Ref. 16) in the discharge plasma of $\mathrm{CO}, \mathrm{H}_{2}$ and $\mathrm{He}$ mixtures, and for $\mathrm{HCN}^{17}$ in the active laser plasma of $\mathrm{HCN}$. The effective vibrational temperature of the stretching modes is estimated from the measurement of the relative intensities, that is $1000-2000$
$\mathrm{K}$ for $\mathrm{HBO},{ }^{14} 700-900 \mathrm{~K}$ for $\mathrm{HCO}^{+},{ }^{16}$ and $1300-1600 \mathrm{~K}$ for $\mathrm{HNC}$. The bending vibrational mode, the $v_{2}$ state, however, is almost thermally populated; $500 \mathrm{~K}$ for $\mathrm{HBO},{ }^{14} 370$ $\mathrm{K}$ for $\mathrm{HCO}^{+},{ }^{16}$ and $230 \mathrm{~K}$ for $\mathrm{HNC}$.

In the present study we have determined the rotational constants in the vibrationally excited states. These allow us to calculate the vibration-rotation constants, i.e., $\alpha_{1}$ $=299.3056(32), \quad \alpha_{2}=-152.4686(26), \quad$ and $\quad \alpha_{3}$ $=335.2003$ (53) $\mathrm{MHz}$ for $\mathrm{HNC}$, and $\alpha_{1}=300.6081$ (111), $\alpha_{2}=-214.6486(85)$, and $\alpha_{3}=238.1349(47) \mathrm{MHz}$ for DNC. The equilibrium rotational constants, taking into account $\alpha_{v}$ 's but not higher order term $\gamma_{v}$ 's, are $45496.7769(45)$ and $38207.7217(105) \mathrm{MHz}$, respectively.

Equilibrium type $\left(r_{e}\right)$ structure is determined from the equilibrium rotational constants of $\mathrm{HNC}$ and $\mathrm{DNC}$ using the conversion factor $505379.05(31) \mathrm{u} \AA^{2} \mathrm{MHz}$. A summary of $r_{e}$ and $r_{s}$ structures is given in Table III. The $r_{e}$ bond length of NC is in good agreement with the $r_{s}$ value: the difference between them is about $0.003 \AA$. However, the $r_{s}-r_{e}$ value of the HN distance is about $-0.01 \AA$,

TABLE II. Molecular constants of HNC and DNC.

\begin{tabular}{lccc}
\hline & HNC & DNC & \\
\hline$B_{0}$ & $45331.9925(20)$ & $38152.9988(16)$ & $\mathrm{MHz}$ \\
$D_{0}$ & $100.144(77)$ & $69.428(62)$ & $\mathrm{kHz}$ \\
$B_{1}$ & $45032.6869(25)$ & $37852.3907(110)$ & $\mathrm{MHz}$ \\
$D_{1}$ & $99.219^{\mathrm{c}}$ & $68.481(398)$ & $\mathrm{kHz}$ \\
$B_{2}$ & $45484.4611(16)$ & $38367.6474[83]^{\mathrm{b}}$ & $\mathrm{MHz}$ \\
$D_{2}$ & $103.231^{\mathrm{c}}$ & $75.352[676]^{\mathrm{b}}$ & $\mathrm{kHz}$ \\
$q$ & $324.340(13)$ & $290.593[17]^{\mathrm{b}}$ & $\mathrm{MHz}$ \\
$q_{J}$ & $-6.17(47)$ & $-6.17^{\mathrm{d}}$ & $\mathrm{kHz}$ \\
$B_{3}$ & $44996.7922(49)$ & $37914.8639(44)$ & $\mathrm{MHz}$ \\
$D_{3}$ & $99.819(181)$ & $69.639(162)$ & $\mathrm{kHz}$ \\
\hline \hline
\end{tabular}

${ }^{a}$ Values in parentheses represent one standard deviation in units of the last digit.

bEstimated value from experimental error.

'From Ref. 10. Fixed in the analysis.

dAssumed value. 
TABLE III. $r_{e}$ and $r_{s}$ structure of HNC (Å).

\begin{tabular}{lll}
\hline & \multicolumn{1}{c}{$r(\mathrm{H}-\mathrm{N})$} & \multicolumn{1}{c}{$r(\mathrm{~N} \equiv \mathrm{C})$} \\
\hline$r_{e}$ & $0.9960643(29)$ & $1.1683506(16)^{\mathrm{a}}$ \\
& $0.996959(1450)$ & $1.168363(226)^{\mathrm{b}}$ \\
& $0.99357(117)$ & $1.16453(28)^{\mathrm{c}}$ \\
& & \\
$r_{s}$ & $0.985884(120)$ & $1.172055(7)^{\mathrm{b}}$ \\
& $0.98607(9)$ & $1.17168(22)^{\mathrm{c}}$ \\
\hline
\end{tabular}

'This work. The uncertainties are derived from one standard deviation in the $B_{e}$ value. The accuracy is estimated to be about two orders worse than the value in parentheses.

'From Ref. 7.

'From Ref. 4.

which is one order of magnitude larger than that of the NC distance. In most cases, the bond lengths of both $r_{e}$ and $r_{s}$ structures have similar values. The anomaly found here strongly suggests the large amplitude bending vibration of HNC molecule.

The difference between $r_{s}$ and $r_{e}$ structure reflects the residual effects of zero-point vibration; the bending vibration tends to shorten the "average" bond length and the anharmonic stretching vibration to lengthen it. If the hydrogen atom is undergoing a large amplitude bending vibration, the difference between the $r_{s}$ and $r_{e}$ structure will have a large negative value, as is found here for the hydrogen-nitrogen distance of HNC. In fact, for HNC the difference between $r_{s}(\mathrm{H}-\mathrm{X})$ and $r_{e}(\mathrm{H}-\mathrm{X})$ is $-0.01 \AA$, which is much larger than those of isoelectronic molecules, $-0.002 \AA$ for $\mathrm{HNN}^{+},{ }^{7}-0.003 \AA$ for $\mathrm{HCN}^{7}{ }^{7}-0.004 \AA$ for $\mathrm{HCO}^{+}, 7$ and $-0.0007 \AA$ for $\mathrm{HBO}^{15}$ these molecules do not have large amplitude vibrations.

The bending potential function remarkably affects an l-type doubling constant. Table IV shows l-type doubling constants of HNC (and DNC) and the isoelectronic molecules. ${ }^{15,16,18-21}$ Generally, not only the "stiffer" bending potential but also the smaller rotational constant will cause the smaller $l$-type doubling constant. Since all molecules in Table IV except HBO (and DBO) have similar rotational constants, the magnitude of the l-type doubling constant

TABLE IV. Comparison of $l$-type doubling constants for isoelectronic molecules $(\mathrm{MHz}){ }^{\text {a }}$

\begin{tabular}{llcc}
\hline & \multicolumn{1}{c}{$H$ species } & \multicolumn{1}{c}{$D$ species } & Ref. \\
\hline $\mathrm{HBO}$ & $181.995(11)^{\mathrm{b}}$ & $144.139(2)^{\mathrm{b}}$ & 15 \\
$\mathrm{HCO}^{+}$ & $211.7659(41)$ & $171.020(11)$ & 16 \\
$\mathrm{HCN}$ & $224.4766(2)$ & $186.1916(3)$ & 18 \\
$\mathrm{HNN}^{+}$ & $254.966(11)$ & $218.084(78)$ & 19,20 \\
$\mathrm{HNC}^{+}$ & $324.340(13)$ & $290.593[17]$ & $\mathrm{c}$ \\
$\mathrm{HOC}^{+}$ & $518.14(58)$ & & 21 \\
\hline
\end{tabular}

"Values in parentheses represent one standard deviation in units of the last digit.

"'B species.

'This work. See Table II. directly reflects the relative "stiffness" of the bending vibration, $\mathrm{HCO}^{+}>\mathrm{HCN}>\mathrm{HNN}^{+} \gg \mathrm{HNC} \gg \mathrm{HOC}^{+}$. This order is also supported by $a b$ initio calculation. ${ }^{22}$ If the contribution of the rotational constant is removed, the resulting "stiffness" of the bending vibration of HBO will probably be close to that of $\mathrm{HCN}$ or $\mathrm{HNN}^{+}$.

The rotational transitions of the isomer $\mathrm{HCN}$ in the vibrationally excited states were observed in the molecular cloud Ori.KL and the circumstellar envelope IRC $+10216 .^{23,24}$ Very recently an interferometric observation was carried out to constrain the location of the vibrationally excited HCN emmision region in IRC $+10216 .^{25}$ The rotational, centrifugal distortion, and $l$-type doubling constants precisely determined in this work would enable the astrophysical detection of $\mathrm{HNC}$ molecule in the vibrationally excited states, as is carried out for the HCN molecule.

\section{ACKNOWLEDGMENTS}

We thank Professor Shuji Saito of Institute of Molecular Science and Dr. Satoshi Yamamoto of Nagoya University for their helpful suggestions in constructing the microwave spectrometer. We are also grateful to Hideki Sugiura and Takaaki Kanno for their assistance in making the instrument. The present study is supported by Grantin-Aid for Scientific Research (Nos. 01470015, 01632512, and 04233107) from the Ministry of Education, Science and Culture.

${ }^{1}$ L. E. Snyder and D. Buhl, Bull. Am. Astron. Soc. 3, 388 (1971).

${ }^{2}$ L. E. Snyder and D. Buhl, Ann. N. Y. Acad. Sci. 194, 17 (1972).

${ }^{3}$ R. A. Creswell, E. F. Pearson, M. Winnewisser, and G. Winnewisser, Z. Naturforsch. 31a, 221 (1976).

${ }^{4}$ E. F. Pearson, R. A. Creswell, M. Winnewisser, and G. Winnewisser, Z. Naturforsch. 31a, 1394 (1976).

${ }^{5}$ R. J. Saykally, P. G. Szanto, T. G. Anderson, and R. C. Woods, Astrophys. J. 204, L143 (1976).

${ }^{6}$ G. L. Blackman, R. D. Brown, P. D. Godfrey, and H. I. Gunn, Nature 261, 395 (1976).

${ }^{7}$ R. C. Woods, Philos. Trans. R. Soc. London, Ser. A 324, 141 (1988).

${ }^{8}$ D. E. Milligan and M. E. Jacox, J. Chem. Phys. 39, 712 (1963).

${ }^{9}$ D. E. Milligan and M. E. Jacox, J. Chem. Phys. 47, 278 (1967).

${ }^{10} \mathrm{C}$. A. Arrington and E. A. Ogryzlo, J. Chem. Phys. 63, 3670 (1975).

$"$ A. G. Maki and R. L. Sams, J. Chem. Phys. 75, 4178 (1981).

${ }_{12}$ J. B. Burkholder, A. Sinha, P. D. Hammer, and C. J. Howard, J. Mol. Spectrosc. 126, 72 (1987).

${ }^{13}$ S. C. Foster and A. R. W. Mckellar, J. Chem. Phys. 81, 3424 (1984).

${ }^{14}$ Y. Kawashima, Y. Endo, K. Kawaguchi, and E. Hirota, Chem. Phys. Lett. 135, 441 (1987).

${ }^{15}$ Y. Kawashima, Y. Endo, and E. Hirota, J. Mol. Spectrosc. 133, 116 (1989).

${ }^{16}$ E. Hirota and Y. Endo, J. Mol. Spectrosc. 127, 527 (1988).

${ }^{17}$ F. C. De Lucia and P. A. Helminger, J. Chem. Phys. 67, 4262 (1977).

${ }^{18}$ A. G. Maki and D. R. Lide, Ir., I. Chem. Phys. 47, 3206 (1967).

${ }^{19}$ W. C. Ho, C. J. Pursell, D. P. Weliky, K. Takagi, and T. Oka, J. Chem. Phys. 93, 87 (1990).

${ }^{20}$ T. J. Sears, J. Chem. Phys. 82, 5757 (1985).

${ }^{21}$ T. Amano, J. Mol. Spectrosc. 139, 457 (1990).

${ }^{22}$ W. P. Kraemer and P. R. Bunker, J. Mol. Spectrosc. 101, 379 (1983).

${ }^{23}$ L. N. Ziurys and B. E. Turner, Astrophys. J. 300, L19 (1986).

${ }^{24}$ R. Lucas, A. Omont, S. Guilloteau, and Nguyen-Q-Rieu, Astron. Astrophys. 154, L12 (1986).

${ }^{25}$ R. Lucas and S. Guilloteau, Astron. Astrophys. 259, L23 (1992). 